Ebisu

Études japonaises

Études japonaises

48 automne-hiver 2012

Naissance d'une revue féministe au Japon : Seitō

(1911-1916)

\title{
Turbulences génériques du roman au sein de la revue Seitō : partage des normes de l'écriture de soi
}

Shifts in the Genre of the Novel in Seitō Magazine: Partial Ownership of Norms in Writing About Oneself

『青鞀』における小説ジャンルの摇らぎ一自分について書くという

規範の分有一

Maiko Odaira

Traducteur : Brigitte Lefèvre

\section{CpenEdition}

Journals

Édition électronique

URL : http://journals.openedition.org/ebisu/640

DOI : $10.4000 /$ ebisu.640

ISSN : 2189-1893

Éditeur :

Institut français de recherche sur le Japon (UMIFRE 19 MAEE-CNRS), Maison franco-japonaise

Édition imprimée

Date de publication : 1 septembre 2012

Pagination : 119-145

ISSN : $1340-3656$

Référence électronique

Maiko Odaira, «Turbulences génériques du roman au sein de la revue Seitō : partage des normes de l'écriture de soi », Ebisu [En ligne], 48 | automne-hiver 2012, mis en ligne le 03 avril 2014, consulté le 20 avril 2019. URL : http://journals.openedition.org/ebisu/640; DOI : 10.4000/ebisu.640 


\section{Turbulences génériques du roman au sein de la revue Seitō \\ Partage des normes de l'écriture de soi}

ODAIRA Maiko 小平麻衣子*

\section{Avant-propos}

Seitō, soit les «Bas-bleus » (septembre 1911-février 1916), première revue au Japon à être entièrement dirigée par des femmes, est bien connue pour son combat en faveur de l'éveil d'une conscience féminine, ses prises de position avant-gardistes, et surtout pour les débats publiés dans ses colonnes, comme ceux sur la chasteté et l'avortement, mais elle est loin de jouir de la même aura sur le plan littéraire, bien qu'à l'origine sa vocation affichée a été la publication de textes littéraires féminins. De fait, beaucoup de nouvelles ou de romans publiés dans Seitō sont confus, tant au niveau de l'intrigue que de l'utilisation des temps et, évalués à l'aune des grandes œuvres littéraires, ces œuvres se rangeraient plutôt parmi les textes d'amateurs.

Les débuts de Seitō en tant que revue littéraire furent le fruit d'un compromis : une manière de pallier l'interdiction faite aux femmes d'avoir une quelconque activité politique. Compte tenu cependant du rôle joué par Seitō dans l'élargissement vers le champ politique de l'activité des femmes, il serait mal avisé de déplorer qu'aucune des auteures qui ont réussi à se faire une place dans le monde littéraire ne soit issue de Seitō. Quoiqu'il en soit, il fallut attendre la création de l'Association pour une nouvelle critique féministe (Shin feminizumu hihyō no kai 新・フェミニズム批評の会)

* Université Nihon 日本大学. 
dans les années 1990 pour que des critiques littéraires se penchent sur la valeur politique des textes. Depuis, un certain nombre d'études ont été réalisées sur la créativité et le contenu critique de ces œuvres, afin d'y évaluer l'état d'avancement du féminisme. Mais peut-on réduire cette littérature à un simple outil politique ? Les auteures de Seitō n'auraient-elles pas, en exprimant quelque chose d'irrépressible en elles et d'inexprimable autrement que par l'écriture, ouvert de nouveaux horizons dans le champ littéraire?

Le recueil publié par le groupe de recherche "Nouvelle critique féministe », Seitōo yomu『青鉴』を読む (Lire Seitō), présente outre des articles sur l'histoire des idées, des études tant sur le versant littéraire que sur la situation de Seitō en tant que média (Shin feminizumu hihyō no kai 1998). Les résultats de ces recherches constituent une avancée incontestable. La partie consacrée à la littérature, c'est-à-dire la première partie, se présente sous la forme d'études critiques des textes en fonction de leur genre : roman, poésie, tanka 短歌 ${ }^{1}$, pièce de théâtre. Une telle classification ne peut que reposer sur une définition tranchée des genres. Or, c'est précisément sur la possibilité d'une telle définition que portent mes doutes car, au sein de la revue Seitō, les frontières génériques étaient fluctuantes et les caractéristiques des textes regroupés sous le terme shōsetsu 小説 (romans et nouvelles) y étaient pour le moins instables ${ }^{2}$. Il était vital pour ces femmes écrivains de définir le genre littéraire dans lequel elles s’inscrivaient, car ce faisant elles se resituaient par rapport aux écrivains masculins qui dominaient alors la scène littéraire. Je souhaite ici non pas m'attarder sur les œuvres en elles-mêmes, mais sur les spécificités formelles et stylistiques des œuvres des contributrices de Seitō, en abordant de manière transversale genre romanesque et genres apparentés pour examiner les flottements et les turbulences génériques suscités par la construction à tâtons d'un récit de soi

1. Par " poésie ", on entend ici la poésie libre, par opposition au tanka, forme poétique traditionnelle ( $w a k a$ 和歌) composée de cinq vers de 5, 7, 5, 7 et 7 syllabes [N.D.L.R.]. 2. Kitada Sachie 北田幸恵 souligne en matière de critique littéraire l'interpénétration et la destruction des genres (Kitada 1998). Elle examine cette question en tant que "processus d'expression qui démonte et reconstruit sans cesse le soi de manière impulsive ", mais plutôt que de nous intéresser au désir des femmes écrivains, nous examinerons ici l'élaboration des normes génériques en un lieu déterminé. 
à partir de procédés narratifs évoluant en fonction des besoins et des impératifs découverts au fur et à mesure par les auteures qui se répondent l'une l'autre. Je propose de distinguer plusieurs étapes dans cette quête identitaire et littéraire : l'affirmation d'un point de vue proprement féminin et l'élaboration progressive d'un récit de soi au féminin, suivis d'une remise en question de la possibilité même du récit véridique de soi, pour réinvestir finalement la fiction dans une passion partagée pour l'écriture ${ }^{3}$. Le tout en l'espace d'à peine six ans.

\section{Construction d'un point de vue féminin dans l'écriture romanesque}

Notons tout d'abord que si les différents genres tels que roman, poésie, tanka, haïku, pièce de théâtre, journal personnel, réflexion personnelle, traduction, etc., figurent dans les colonnes de Seitō, cela ne signifie pas pour autant que chaque genre était représenté dans chaque numéro de la revue. Le genre auquel appartenait un texte était simplement indiqué dans la table des matières après le titre de chaque ouvre. Cette classification reflétait souvent l'inspiration du moment de la rédactrice en chef.

\section{Les premières fictions d'inspiration autobiographique}

La période allant de la première à la deuxième année se caractérise par une livraison importante de "romans ${ }^{4}$ ». Ces « romans " se caractérisent par la présence de personnages fictifs librement créés à partir de modèles réels dans des récits écrits à la troisième personne, même lorsque l'auteure s'inspirait de sa propre vie. La narration se focalisait sur la vie intérieure tant des personnages féminins que masculins. Sont représentatifs de cette catégorie

3. Ci-après, les titres et les citations se réfèrent, sauf mention précise, à des textes publiés dans Seitō. Entre parenthèses, nous donnons le genre indiqué dans le sommaire du numéro de Seitō, suivi du mois et de l'année de parution. Pour les commentaires critiques sur ces textes, voir les études du groupe Raichō kenkyūkai (2001).

4. Cette catégorie shōsetsu regroupe des récits fictionnels de tailles variées, qui vont de la nouvelle au roman long. Elle est traduite ici par commodité par le terme " roman " [N.D.L.R.]. 
les romans de Kiuchi Tei 木内錠 par exemple, qui a publié successivement Yügeshō 夕化粧 [Maquillage du soir] (roman, nov. 1911), Sasurai さすらひ [Errance] (roman, janv. 1912), Tanin no ko 他人の子 [L'Enfant de l'autre] (roman, fév. 1912), et Rōshi 老師 [Le Vieux maître] (sept. 1912). Ces quatre romans, bien qu'écrits les uns à la suite des autres, ne présentent pas de liens entre eux, car chacun met en scène des personnages différents dans un environnement particulier. Parmi les œuvres de la deuxième catégorie, on peut citer Karekusa 枯草 [Herbes fanées] (roman, fév. 1912) et Antōo 暗闘 [Une sourde hostilité] (roman, avril 1912) d'Iwano Kiyo 岩野清 et Kyaku 客 [L'Invité] (roman, juin 1912) d'Ogasawara Sada 小笠原さだ. Herbes fanées dépeint les souffrances de l'héroïne, Sayoko, qui doit supporter seule tous les soucis ménagers, Une sourde hostilité a pour thème les frictions entre une épouse, Kimiko, et l'ancienne maîtresse de son mari.

Pour ces deux œuvres, on ne peut deviner à partir de la seule lecture du texte si l'auteure s'est inspirée de faits personnels réels. La consultation des documents biographiques d'Iwano Kiyo et des romans d'inspiration autobiographique (shishōsetsu 私小説) de son mari Iwano Hōmei 岩野泡鳴 permet de le vérifier. De plus, le point de vue du personnage principal est relativisé dans Une sourde hostilité car l'auteure dresse non seulement le portrait psychologique de l'héroïne, Kimiko, mais aussi ceux de son mari et d'Otori, sa première femme. Ces auteures avaient évidemment beaucoup appris des romans des auteurs masculins qui les avaient précédés et de ce fait leur servaient encore de modèle. Toutefois, on attendait d'elles qu'elles fassent preuve dans leur vie comme dans leurs œuvres d'une valeur jugée intrinsèquement féminine à savoir l'empathie. Il est dès lors peu surprenant de constater dans ces premiers romans une attention particulière portée aux personnages masculins car, les critiques littéraires l'ont souvent fait remarquer, les conditions pour que les femmes écrivent sur elles-mêmes n'étaient pas encore réunies.

\section{Le poids grandissant des personnages féminins}

À partir de la troisième année, on constate un changement général dans la manière d'écrire. Même si elles continuent à écrire des romans à la troisième personne, la plupart des auteures se focalisent dorénavant sur un personnage féminin. Par exemple, on peut affirmer d'après les faits relatés que les romans Uranai 占w [Divination] (roman, sept. 1913) d'Iwano Kiyo 


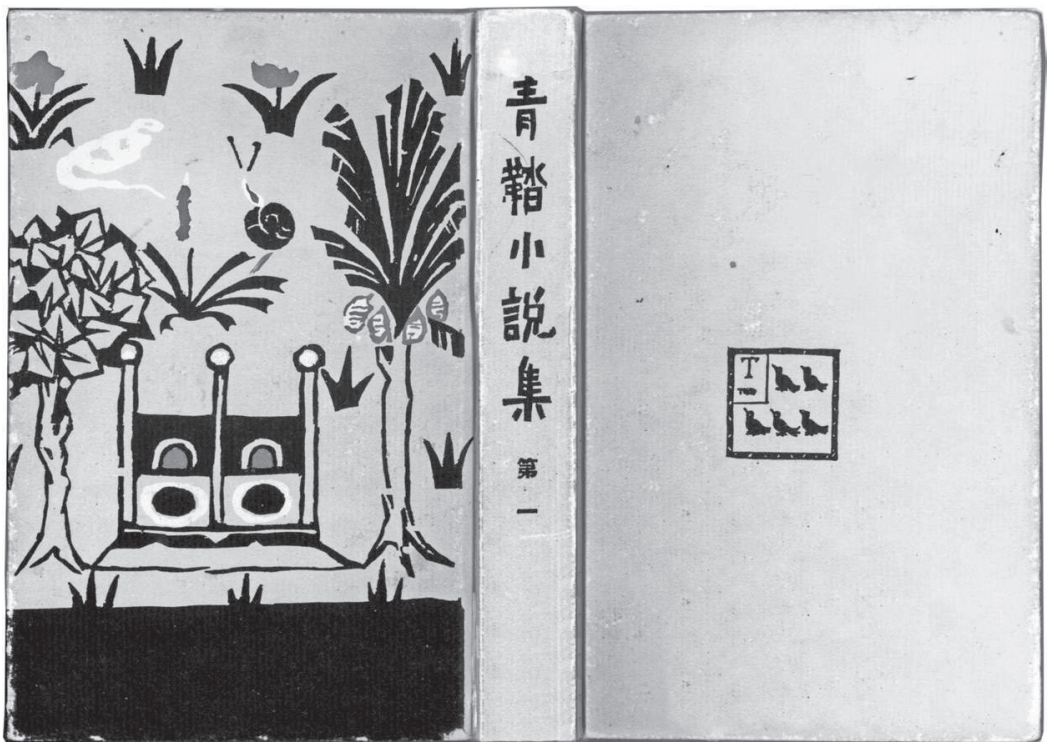

Fig. 1. Premier recueil de romans Seitō shōsetsu-shū dai ichi 青鞜小説集第一 publié par Seitōsha (février 1913), couverture dessinée par Ogasawara Sada.

et Machiwabi 待ち侘び [Attendre avec impatience] (roman, oct. 1914) de Hayashi Chitose 林千歳 s'inspirent directement de l'expérience personnelle des auteures, et qu'ils sont en cela exemplaires de la tendance nouvelle visant à créer des romans désormais centrés sur des hérö̈nes inspirées de l'auteure. Nyobō hajime 女房はじめ [Début de la vie d'épouse] (roman, janv. 1915) d'Ueno Yō 上野葉, met en scène les débuts de la vie conjugale de la jeune Teruko après une période pendant laquelle le couple a fait chambre à part par respect pour sa virginité. Le texte est écrit à la troisième personne mais la narratrice privilégie dans sa narration le cheminement intérieur de Teruko. Rares sont les passages descriptifs pris en charge par la narratrice pour parler des autres personnages, ce qui finalement donne l'impression au lecteur que Teruko et la narratrice ne font qu'une. Ueno Yō ayant préalablement écrit des textes critiques sur le mariage, il ne fait aucun doute que ce roman réécrit à la première personne pourrait passer pour un article critique de cette même auteure. 
On peut porter un avis défavorable sur la technique romanesque d'Ueno Yō, mais il faut aussi se rappeler ces mots d'Iwano Kiyo écrits dans Omotte iru koto 思ってるる事 [Ce que je pense] (Réflexions personnelles, mai 1914) : " J'aimerais endormir cet esprit froid qui m’observe, moi, de l'extérieur et me critique, pour, ne serait-ce qu'une fois, même fugitivement, libérer vraiment mon moi ». En cherchant à se détacher du processus laborieux de prise de conscience de soi en vogue à l'époque moderne, Iwano veut aussi se défaire d'une préoccupation liée à la place sociale accordée à la femme. La pratique de la méditation et de la réflexion, en développant une vue surplombante de soi et une faculté d'autocritique, devait permettre aux femmes de se distancier des soucis domestiques pour conserver leur sang froid et réagir en chaque occasion avec calme et pondération. De la même manière, dès lors qu'une romancière moderne adoptait ce dispositif faisant du narrateur une instance qui surplombe l'ensemble de l'œuvre, même si elle mettait en scène un personnage qui lui ressemblait, la position de son personnage féminin s'en trouvait forcément relativisée. La question se posa alors de savoir comment mettre en avant le point de vue et les opinions des femmes en dépit de ce processus de distanciation en vogue dans le monde littéraire ? Les auteures ont été confrontées à la question de savoir comment accorder écriture romanesque et féminisme. Cette recherche ne se manifeste d'ailleurs pas seulement dans le roman, elle est également sensible dans les va-et-vient effectués d'un genre à l'autre car, à l'instar d'Iwano Kiyo et d'Ueno Yō, rares ont été les membres de Seitō à n'écrire que des romans. À supposer d'ailleurs que certaines se soient spécialisées dans ce genre, étant elles-mêmes en même temps des lectrices de Seitō, elles ne pouvaient pas ne pas être influencées par le travail effectué par d'autres dans d'autres domaines.

\section{Réactions de la revue Seitō aux critiques extérieures}

À partir de sa troisième année d'existence, la revue publie de plus en plus de textes interpellant la conscience des hommes, qui tendent ainsi à construire une position antagoniste avec le monde extérieur. Le mouvement de la "femme nouvelle » était caricaturé dans les journaux et revues qui se gaussaient d'autant plus des auteures de Seitō qu'ils n'avaient qu'une vue superficielle des questions traitées, ne retenant par exemple que la visite dans le quartier de prostitution, la consommation d'alcool ou encore la 
défense par certaines de l'amour libre. La revue Seitōa dû, pour lutter contre ces préjugés et ces malentendus, clarifier progressivement ses objectifs.

Ainsi que l'écrit Itō Noe 伊藤野枝 dans Kono goro no kansō 此の頃の感想 [Réflexions de ces derniers temps] (sans précision générique, fév. 1913) : "Sur divers points, c'est à un éveil sincère de la conscience des hommes que j'appelle. Je souhaiterais même qu'il précède l'éveil de nos consciences à nous les femmes. " Elle souligne ainsi que pour en finir avec l'oppression des femmes, il faudrait que les hommes, plus encore que les femmes, commencent à évoluer dans leur manière de penser. Ces argumentaires sont présentés à la première personne. Nous n'examinerons pas ici le contenu des critiques publiées, mais notons qu'elles présentent en commun le fait que l'auteure y nomme expressément l'interlocuteur ou l'interlocutrice faisant l'objet de son attaque ou de sa contre attaque.

De même, pour ne parler ici que des membres importants, Hiratsuka Raichō 平塚らいてう s'oppose à Yamaji Aizan 山路愛山去 dans Henshūshitsu yori 編集室より [Note de la rédaction] (mars 1913) et relance ensuite le débat par la critique qu'elle fait des romans de Tamura Toshiko 田村俊子 dans Morita Sōhei shi ni 森田草平氏に [À M. Morita Sōhei] ${ }^{6}$ (aucune précision générique dans ce numéro, août 1914) ; Iwano Kiyo poursuit ce combat en publiant Kaetsu Taka joshi ni 嘉悦孝女史に [À Mme Kaetsu Taka ${ }^{7}$ (Note de la rédaction, janv. 1914); Itō Noe publie une série de textes incisifs avec Mushanokōji shi ni 武者小路氏に [Lettre à M. Mushanokōji] (Note de la rédaction, janv. 1914), Yonda mono kara 読んだものから [Notes inspirées de mes lectures] (réflexions récentes, juin 1914), texte dans lequel elle critique Nakazawa Rinsen 中沢臨川 ${ }^{8}$, Shimoda Jirö shi ni 下田次郎氏に

5. Yamaji Aizan (1865-1917) historien, critique et penseur, il est le premier à comparer Ogyū Sorai 荻生徂徠 à Machiavel dans un ouvrage en 1893. Il fonde en 1905 le parti socialiste d'État (Kokka shakaitō 国家社会党) qui s'éteint dès 1906 [N.D.E.].

6. Morita Sōhei (1881-1949), romancier connu pour son livre Baien 煤煙 (Suie) qu’il a tiré de l'affaire de suicide d'amoureux raté impliquant la fondatrice de Seitō, Hiratsuka Raichō, et connue sous le nom de Shiobara jiken 塩原事件 [N.D.E.].

7. Kaetsu Taka (1867-1949), fondatrice en 1903 de l'École de commerce pour filles, devenue depuis 2001 université Kaetsu [N.D.E.].

8. Nakazawa Rinsen (1878-1920), proche de Kunikida Doppo 国木田独歩 et d'Osanai Kaoru 小山内薰, publie de nombreux articles dans Chūō kōron 中央公論 à partir de 1912, après avoir publié un article sur Tolstoï [N.D.E.]. 
[Lettre à M. Shimoda Jirō] ${ }^{9}$ (réflexions personnelles, juillet 1914), Shimoda Utako joshi e 下田歌子女史へ [À Mme Shimoda Utako] ${ }^{10}$ (critique, oct. 1914), et enfin Matsumoto Gorō shi ni kotau 松本悟郎氏に答ふ [Réponse à M. Matsumoto] (sans précision générique, nov. 1914). Chaque fois Itō s'en prend à des auteurs et des critiques masculins mais aussi féminins, tous défenseurs de l'idéologie ryōsai kenbo 良妻賢母 ( (bonne épouse, mère avisée ") et de l'image traditionnelle de la femme vertueuse. On peut dire que c'est précisément dans et par ce mouvement de différenciation et d'opposition vis-à-vis de la société que le groupe Seitō s'est constitué. C'est en redessinant les frontières entre l'extérieur et l'intérieur qu'il forgeait peu à peu son identité. L'évolution de l'écriture romanesque vers une focalisation sur un personnage féminin est une manifestation concrète de ce processus de différenciation.

\section{2. Élaboration du récit de soi et prise de distance avec le roman}

Rien de surprenant à ce qu'apparaissent, à ce stade, des textes écrits à la première personne, qui relatent l'expérience personnelle des auteures. Je propose de nous arrêter ici sur les textes dans lesquels ces femmes expriment leurs impressions et leurs sentiments sans se préoccuper de conclure leur récit ou de lui donner une cohérence logique.

\section{Premiers récits à la première personne}

Ichinenkan 一年間 [Une Année] (sans précision générique, fév. 1913), récit à la première personne de Hiratsuka Raichō, a défrayé la chronique en retraçant les amours homosexuelles de l'auteure avec Otake Kōkichi (Beniyoshi) 尾竹紅吉. Dōyō 動摇 [Troubles] (sans précision générique,

9. Shimoda Jirō (1872-1938), auteur d'une thèse universitaire puis du premier ouvrage sur l'éducation des filles (Joshi kyōiku 女子教育, 1904), il fonda également la Société japonaise pour l'éducation des filles.

10. Shimoda Utako (1854-1936), fondatrice de l'Association impériale des femmes (Teikoku fujin kyōkai 帝国婦人協会) en 1899, elle fut professeure au lycée pour les filles de l'aristocratie (Kazoku jogakkō 華族女学校) fondé en 1885 [N.D.E.]. 


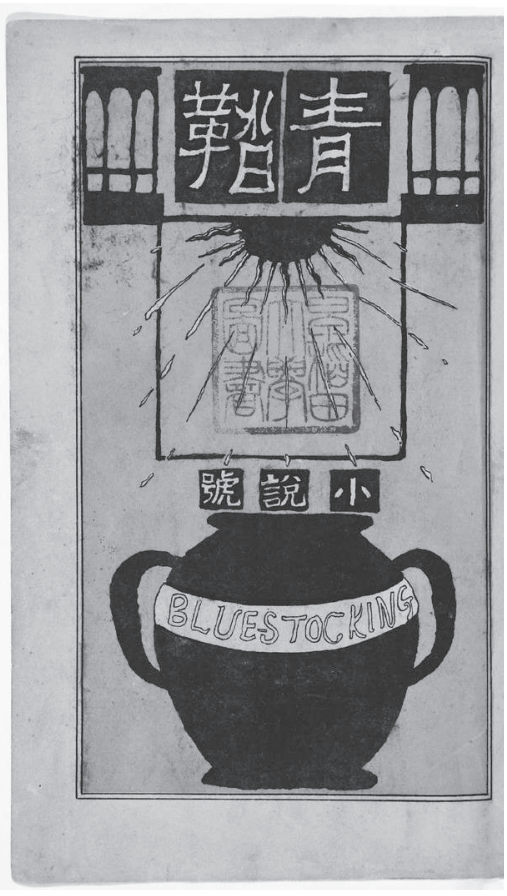

Fig. 2. Couverture de Seitō, avril 1912, consacré aux romans, avec un dessin d'Otake Kōkichi (Beniyoshi). Bibliothèque de l'université Waseda.

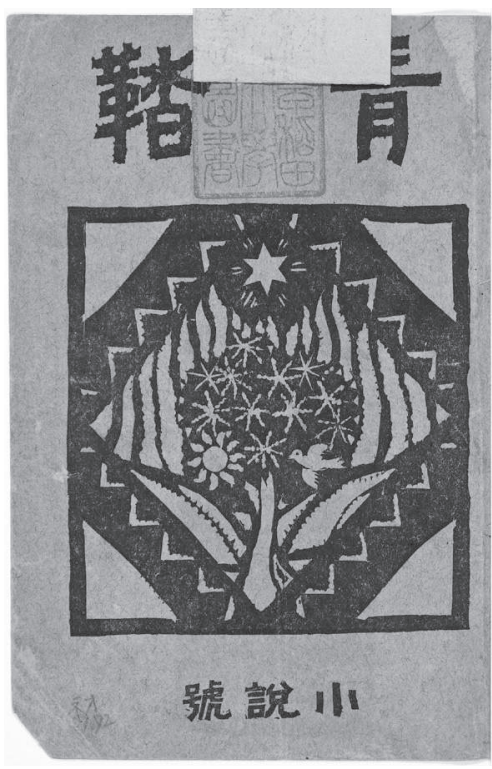

Fig. 3. Couverture de Seitō, avril 1914, consacré aux romans, avec un dessin d'Okumura Hiroshi 奥村博. Bibliothèque de l'université Waseda.

août 1913) d'Itō Noe, relate l'impasse amoureuse dans laquelle l'auteure s'est trouvée avec Kimura Sōta 木村壮太 qui s’était épris d'elle sans savoir qu'elle vivait avec Tsuji Jun 辻潤 et était enceinte de lui. Écrire une expérience personnelle à la première personne peut sembler évident, mais il leur fallait en réalité dissocier le simple fait d'écrire sur soi, du procédé narratif consistant à écrire un récit à la première personne. Ces deux processus sont radicalement différents. Nous avons vu précédemment que les expériences personnelles étaient jusqu'alors relatées à la troisième personne. On peut en déduire que les auteures, en faisant désormais coïncider thème (autobiographique) et procédé narratif (récit à la première personne), voulaient expurger de leur écriture toute objectivation réflexive, c'est-à-dire toute mise à 
distance par rapport à soi, afin d'édifier une posture d'affirmation subjective de soi. Ainsi, le poème Sozorogoto そぞろごと [Flânerie] paru dès le premier numéro de la revue, est-il resté gravé dans les mémoires car la poétesse Yosano Akiko 与謝野晶子 y clamait : "J'écris toujours à la première personne. / Je suis une femme. / Je n'écris qu’à la première personne. / Je suis moi. " Pour autant, l'apparition d'une prose écrite à la première personne prendra, ainsi qu'en témoignent les oscillations des frontières génériques au sein de la revue Seitō, plus de temps qu'on ne l'avait imaginé.

C'est pourquoi, même lorsqu'il s'agissait d'un " récit » à la première personne, il n'était pas construit de manière à donner une vision en perspective des événements passés. En principe, le personnage en charge du " je " en train de raconter des événements passés, dispose de beaucoup plus d'informations que n'en avait au moment des événements le « je », objet du récit ; ce décalage permet au narrateur de redonner rétrospectivement du sens au passé. Toutefois Une année et Troubles retracent divers moments dans le passé qui font ressurgir chez les auteures les émotions successives éprouvées en ces instants, sans que celles-ci ne cherchent à reconstituer une appréhension cohérente des événements tels qu'ils pouvaient apparaître dans le présent de l'écriture (Tokunaga 2010). Cela tient bien sûr au fait que, dans ces deux romans, les émotions sont peintes telles qu'elles ont été ressenties sur le moment dans l'ignorance de l'avenir. D'un point de vue narratif, cette confusion, ou plus exactement cette manière d'écrire sans recul, correspond à un rejet délibéré que l'on pourrait assimiler à une volonté d'éradiquer toute attitude critique fondée sur la hiérarchisation du présent et du passé.

\section{Distinction entre fiction et récit de soi}

Les auteures qui ont adopté cette forme d'écriture romanesque à la première personne ont alors éprouvé la nécessité d'affirmer avec véhémence qu'elles n'écrivaient pas des "romans ". Ainsi, dans Une Année, Raichō écrit : "Ceci n'est pas un roman. Je n’ai jamais pensé que c’en était un. (...) J'insiste vraiment sur le fait que je n'ai pas pris la plume avec le dessein d'écrire un roman."

De même, dans Troubles, Itō Noe prend la précaution de préciser le pacte de lecture qu'elle souhaite contracter avec ses lecteurs : "Ceci n'est pas un roman. Je veux que mes lecteurs lisent simplement ce texte comme le récit véridique de faits réels. » Dans Aru hi 或る日 [Un Jour] (roman, sept. 1913) 
Katō Kazu 加藤籌 raconte à la troisième personne, par le biais d'un personnage interposé, Fujiko, la mort de son propre enfant, mais elle clôt son récit en ces termes : «Je ne sais pas si ce que j'ai écrit peut être considéré ou non comme un roman. Peu m'importe d'ailleurs. À dire vrai, je n'ai fait qu'écrire les événements d'un jour tels qu’ils se sont vraiment passés ", reconnaissant ainsi qu'il s'agissait de sa propre expérience.

Si elles ressentent alors la nécessité de préciser de manière délibérée que leurs textes ne sont pas des romans, c'est précisément parce qu'ils ressemblent beaucoup à ce qu'elles appelaient jusqu'alors " roman ». Jusque-là, pour déterminer si l'œuvre en question relevait ou non du genre romanesque, les éditrices se fondaient sur le pacte de lecture et la structure du texte plus que sur la véracité des faits. Était considéré comme un essai critique tout texte qui au moyen d'une argumentation étayée de preuves tendait à conduire le lecteur vers une conclusion. Sinon il s'agissait d'un roman, autrement dit, d'un texte dans lequel l'auteure s'attachait avant tout à peindre des scènes et des émotions et à structurer la temporalité à l'intérieur du récit. Le refus catégorique de certaines que leur texte soit classé dans le genre romanesque montre, comme on pouvait s'y attendre, qu'elles ont dû redéfinir le roman comme genre fictionnel, pour s'écarter précisément de ce modèle et privilégier la vérité. Par leur refus, elles retraçaient les frontières génériques au sein de la revue Seitō. Néanmoins, en clamant haut et fort que leurs écrits n'étaient pas des romans, elles se gardaient bien de définir le genre auquel ces écrits devaient être rattachés. Bien qu'elles relataient des faits tenus pour véridiques, elles maillaient leurs textes de souvenirs et d'anachronismes contredisant toute exigence logique, et s'autorisaient à ne pas suivre la chronologie des événements. Cela parce qu'elles préféraient exprimer les différentes facettes des émotions ressurgies pendant l'écriture plutôt que de retracer fidèlement une vérité objective. Elles privilégiaient désormais la vérité de la personne qui écrit.

Si l'on tient compte du fait que chez les auteurs masculins, la confession d'expériences personnelles était rattachée au genre romanesque, retracer de cette manière les frontières des genres littéraires était aussi pour ces auteures une manière de définir leur propre territoire en le démarquant de celui des hommes. À l'époque, c'était le genre romanesque et non la poésie et les waka 和歌 qui dominaient la scène littéraire, et lorsque les auteurs ou les éducateurs encourageaient les femmes à écrire, c'était en leur conseillant d'écrire ce qui, à leur sens, leur convenait le mieux, qui des 
waka, qui des essais, tout à l'exception des romans. Sans doute les hommes répugnaient-ils à laisser les femmes écrivains entrer dans un territoire qu'ils occupaient eux-mêmes et les guidaient-ils inconsciemment vers des territoires périphériques (Odaira 2008 : 111-158). Les contributrices de Seitō, en refusant le roman, ne faisaient que confirmer cette mise à l'écart dans un espace littéraire périphérique, en marge du courant romanesque dominant. Leur attitude peut être interprétée comme une forme de résignation et de soumission aux règles établies par les hommes pour les reléguer au second rang. Cependant, en tant que posture antagonique, cette attitude revêt une autre signification : il est difficile de savoir jusqu'à quel point elles étaient conscientes de ce qu'elles faisaient, mais le choix d'un genre littéraire d'opposition a joué un rôle décisif dans l'édification d'une position autre, à savoir celle de la «femme».

Entrant dans cette dynamique, les contributrices de Seitō ont pris pour matériaux leurs propres expériences et ont adopté cette norme du récit de soi, imposant que la narratrice en charge du récit soit facilement identifiable par les lectrices comme étant l'auteure. La norme s'est ensuite imposée à l'ensemble de la production littéraire de Seitō, ce qui explique pourquoi certaines contributrices importantes de la revue ont critiqué l'usage de pseudonymes. Par exemple, dans Meguro kara 目黒から [Depuis Meguro] (sans précision de genre, fév. 1913), Iwano Kiyo s’adresse à Hiratsuka Raichō en l'appelant par son vrai prénom "Haruko ", critiquant ainsi implicitement l'usage du nom de plume "Raichō ". Raichō quant à elle critiqua aussi sévèrement le recours d'Okada Yachiyo 岡田八千代 à ce procédé, lorsque prétendant renoncer à écrire, celle-ci présenta Date Mushiko 伊達虫子 comme sa remplaçante dans les notes de la rédaction (Henshüshitsu yori 編集室より) (avril 1914). Raichō s’insurge contre ce subterfuge : «Je n’apprécie pas beaucoup les paroles et les attitudes de ceux et celles qui s'introduisent dans les cénacles littéraires en changeant de nom " (Yonda mono no hyō to saikin zatsukan 読んだものの評と最近雑感 [Critiques de lectures et impressions récentes], mai 1914), signifiant par là que l'usage d'un pseudonyme, ou d'un nom de plume, rendait caduque la confession autobiographique d'un auteur, en la nimbant d'un voile de fiction ce qui, dans certains cas, pouvait éroder la responsabilité de l'écrivain. En effet, le lecteur ne sait plus qui écrit, ce qui s'oppose au principe prévalant selon lequel le sujet décrit dans le texte devait coïncider avec l'auteur en personne. 


\section{Recours à la forme épistolaire}

En même temps, apparaissent des textes qui prennent la forme d'une lettre adressée à une personne précise, tout particulièrement dans les kansō 感想 (réflexions personnelles) et les hihyō 批評 (critiques) qui ne relèvent pourtant pas du genre épistolaire. Celle-ci n'est plus un interlocuteur ou une interlocutrice pris à partie dans une polémique comme on l'a vu précédemment, mais un tiers choisi parmi les proches de l'auteure. Iwano Kiyo s'adresse ainsi à "Haruko " (Hiratsuka Raichō) dans Depuis Meguro, Itō Noe s'adresse à «R. » (Raichō) dans Somei yori 染井より [Depuis Somei] (sans précision générique, juillet 1913), Iwano Kiyo s'adresse à " Mme I. " (Itō Noe), dans Kita no kōgai yori 北の郊外より [Depuis la banlieue nord] (sans précision générique, août 1913), Kobayashi Katsu 小林哥津 s’adresse à «Mlle Raichō» (Hiratsuka Raichō) dans Botan bake o yonde牡丹刷毛を読んで [Après avoir lu Pinceau pivoine ${ }^{11}$ ] (sans précision générique, oct. 1914), et Hiratsuka Raichō s'adresse à "Noe " (Itō Noe) dans Onjuku yori 御宿より [Depuis Onjuku] (lettre, nov. 1914), pour ne citer que quelques exemples parmi de nombreux autres.

Compte tenu du lien direct entre le sujet « je » du roman et l'auteure en personne, il est permis de penser qu'en désignant explicitement leur destinataire, les auteures souhaitaient se protéger et éviter les malentendus. Notons que l'existence de ce lien direct avait aussi pour effet d'accentuer la conscience des contributrices de faire partie d'un même groupe constitué comme «sujet " face au monde. Raichō écrit dans Saikin no kansō 最近の 感想 [Réflexions récentes] (réflexions personnelles, juin 1914) : «Un jour j'en suis venue à me soucier de l'impact social de mes écrits ». Les propos de Seitō étant repris et abondamment critiqués dans les journaux et les grandes revues de l'époque, ces discours dépassaient largement le cadre de causeries plaisantes entre membres de cette revue. Dans ces conditions, et puisqu'on ne pouvait clairement délimiter le public qui achetait ou lisait la revue, les contributrices de Seitō, dans le but de se protéger peu ou prou contre les

11. Essai de l'actrice Matsui Sumako [N.D.T.]. À propos de cette actrice, voir les textes de Christine Lévy et de Niimura Yōko dans le même dossier. Le " pinceau pivoine " (botan bake) est un accessoire de maquillage, en particulier des gens du spectacle, servant à appliquer la poudre blanche (白粉 oshiroi) sur le visage et la nuque des comédiens et des danseurs [N.D.L.R.]. 
réactions imprévues de l'extérieur, prenaient la précaution de souligner à l'aide de cette adresse bien ciblée que leurs textes énonçaient des opinions destinées à des personnes adhérant à l'esprit de la revue.

Cependant, précisément pour cette même raison, ces textes n'étaient absolument pas des lettres à caractère privé. S'il s'était agi de simples commentaires de la rédaction, ces textes auraient pu figurer en arrière-plan, à la fin de la revue, mais ils étaient au contraire placés bien en évidence dans les pages principales et constituaient les grands titres de la revue, ce qui donne à penser qu'ils tenaient lieu de déclarations publiques de la revue Seitō. Les destinataires, même lorsqu'elles n'étaient désignées que par leurs initiales, étaient aisément identifiables par les lectrices régulières de la revue ${ }^{12}$. En même temps, à travers cette personne désignée, les auteures s'adressaient à la communauté de lectrices qui partageaient les mêmes intentions. En ce sens, n'importe qui pouvait s'identifier à la personne désignée comme destinataire, car s'il s'était agi de textes ne visant que la personne à qui ils étaient adressés nominalement, il aurait suffi d'envoyer une lettre à cette personne. En réalité, ces textes ainsi "destinés " créaient une relation de complicité avec les lectrices, à la manière de relations épistolaires entre des personnes qui ne se connaissent pas particulièrement.

Cette évolution n'est pas le fruit d'une décision unilatérale et ponctuelle, elle résulte d'un processus graduel. Itō Noe est une de celles qui avaient le mieux intériorisé ces nouvelles normes. Après Troubles, déjà cité, elle a publié Wagamama わがまま [Égoïste] (roman, déc. 1913) et Shuppon 出奔 [Fuite] (roman, fév. 1914), romans à la troisième personne ayant tous pour héroïne Toshiko. Elle a publié ensuite Itoko ni 従妹に [À ma cousine] (lettre, mars 1914) écrit sous la forme d'une lettre, Madoi 惑ひ [Errance] (roman, avril 1914), Esu sensei ni S 先生に [Au professeur S.] (lettre, juin 1914), Isho no ichibu yori 遺書の一部より [Extrait de testament] (roman, oct. 1914), qui étaient tous des textes épistolaires écrits à la première personne. Notons qu'un tel va-et-vient entre romans et lettres implique aussi une alternance entre troisième et première personne. Dans ses romans à la troisième personne, Itō Noe raconte des événements directement autobiographiques :

12. L'utilisation d'initiales et de noms de plume était un phénomène très répandu en d'autres lieux que Seitō. Mon but est ici d'examiner l'évolution de cette fonction, au sein même de Seitō. 
comment elle en est venue à haïr son mariage forcé, à monter à la capitale, etc. Ses textes épistolaires sont des plaidoyers pour son mode de vie. D'un point de vue formel, elle refuse le roman, et si ces lettres s'adressent à deux destinataires spécifiques, $A$ ma cousine et $A$ u professeur $S$., c'est pour éviter d'être couverte d'opprobre par l'ensemble de la société. Ainsi Itō Noe représente-t-elle parfaitement Seitō, non seulement par ses opinions, mais également par les formes littéraires qu'elle a adoptées.

\section{Mise à distance du récit véridique et du récit de soi}

\section{Évolution de la fonction et de l'utilisation des initiales}

Le schéma de communication va connaître une nouvelle transformation à partir de la cinquième année. Nous l'avons dit, les auteures avaient jusque-là recours à des initiales pour désigner la destinataire de leur texte, et celle-ci étant une éminente collaboratrice de Seitō, les lectrices savaient immédiatement de qui il s'agissait. Or, à partir de la cinquième année, il devient impossible de savoir de qui sont ces initiales, lesquelles ne sont plus utilisées pour interpeller une personne précise.

Satomi Matsuno 里見マツノ, dans un passage de Zakkichō yori 雑記帳より [Extrait de mes cahiers] (sans précision générique, janv. 1915), a recours à des initiales dans la description de la promenade d'un jeune garçon avec deux femmes plus âgées que lui. Arita Sei 有田勢伊, dans $\bar{E}$ to $K \bar{e}-k o \mathrm{~A} と \mathrm{~K}\}$ [A. et K.] (roman, mars 1915), présente, par le biais d'une discussion entre un jeune homme A. et sa fiancée K., les différences de point de vue entre homme et femme sur la chasteté. C'est un récit à la troisième personne, écrit dans un style particulier à partir de propos rapportés à la forme indirecte.

Par ailleurs, Shinjitsu no kokoro yori 真実の心より [D’un cœur sincère] (roman, juil. 1915) de Hamano Yuki 浜野雪 dresse le portrait d'une femme divorcée vivant avec son enfant tout en entretenant une relation amoureuse avec I., lequel vit avec une autre femme. Empêtrée dans cette situation inextricable, elle se morfond, incapable d'avoir selon elle une relation sincère avec elle-même et avec I., si bien qu'elle décide finalement de rompre pour vivre une relation purement amicale avec lui, mais elle ne cesse ensuite de se reprocher sa lâcheté. Chihara Yoshi 千原与志, dans Shojosaku 処女作 [Première Euvre] (roman, avril-juil. 1915), met en scène une femme "Y. » dont s'éprend un pasteur marié. Ce dernier va 
jusqu'à envisager le divorce pour commencer une autre vie avec elle, mais Y. mettant en doute son amour pour elle choisit de mettre un terme à leur relation. Si l'analogie entre Y. et l'auteure (Chihara) Yoshi saute aux yeux, en revanche, on ne sait pas à qui renvoient les initiales utilisées pour les autres personnages.

Les principaux membres de Seitō avaient pris des mesures en octobre 1913 pour créer un groupe de soutien avec pour objectif premier de remédier aux difficultés financières de la revue, ce qui a eu pour conséquence l'afflux d'une nouvelle vague de collaboratrices, différentes des fondatrices, qui vinrent augmenter les effectifs. Ensuite, après le remplacement à la rédaction de Hiratsuka Raichō par Itō Noe (janv. 1915), l'ancien système d'organisation qui établissait une hiérarchie entre les membres de l'association Seitōsha 青鞜社 ayant été supprimé, il devint plus facile pour les nouvelles collaboratrices de publier leurs œuvres. La publication des textes de nouvelles recrues a ainsi augmenté à partir de la cinquième année, avec pour conséquence pour les lectrices de ne plus pouvoir décrypter qui se cachait sous les initiales, faute de renseignements sur ces nouvelles collaboratrices, alors que la vie des fondatrices était connue de toutes grâce aux "notes de la rédaction ". Même lorsque, pour l'auteure, les initiales désignaient une personne réelle, les lectrices ne pouvaient plus faire le lien avec la dite personne. Au final, on peut avancer que l'évolution de la fonction des initiales n'est pas tant liée à un changement important des normes d'écriture qu'à sa systématisation.

En d'autres termes, ainsi que le déplore ci-après Shibata Kayo 柴田かよ dans Mino yori 美濃より [Depuis Mino] (1913), plus les auteures veulent écrire la " vérité " dans Seitō, et plus il leur devient difficile de le faire :

Aujourd'hui, même dans un petit poème ou un texte court, je reste très attachée à la description de la vérité. (...) Ma vue est obscurcie par cette atmosphère oppressante dans laquelle la femme est confinée et qui lui dénie toute liberté, ainsi que par cette foule de gens vulgaires et sans idées. (...) Mais dans la situation dans laquelle je me trouve, on ne tolèrerait absolument pas que je manifeste mes sentiments.

Ainsi Shibata à qui l'on avait demandé d'écrire pour Seitō écrivait-elle, au lieu d'envoyer une œuvre de fiction, les raisons pour lesquelles elle ne pouvait pas le faire. Mais, si elle craignait d'être réprimandée par un entourage très conventionnel, c'est bien parce qu'elle se faisait une haute idée de l'écriture de la "vérité ", comme témoignage de faits réels vécus 
par elle-même et expression sincère de ses sentiments et de ses émotions. Ainsi, la norme selon laquelle il fallait écrire ses propres expériences de manière à ce qu'elles soient reconnues s'intériorisait-elle plus profondément encore. C'est pourquoi Shibata redoutait tant que ses écrits pour Seitō viennent à être connus de son entourage. Le cours paisible de sa vie quotidienne s'en trouverait bouleversé. Il est vrai aussi que les relations décrites dans D'un cour sincère et Première Euvre heurtent quelque peu les bienséances.

\section{Retour des pseudonymes et création de personnages éponymes}

Autrement dit, la notation d'initiales a un tout autre but que précédemment : désormais les initiales permettent de dissimuler un peu l'auteure et les personnes concernées par son récit, sans pour autant mentir ou voiler la vérité. On peut établir un parallèle entre cette nouvelle tendance et le retour chez les fondatrices à l'usage des pseudonymes pourtant critiqués au début. Plusieurs romans prennent pour titre le nom d'un personnage éponyme : Yamanoi Mineko 山の井み衩子, nom féminin formé à partir d'une composition délibérée de mots élégants, Nanako 奈々子, qui signifie aussi « sans nom » de Hasegawa Shigure 長谷川時雨. De même, dans le texte publié en feuilleton dont nous avons parlé précédemment, Chihara Yoshi change de signature en cours de route pour adopter celle de Sakurako さくら子. Même Itō Noe qui, on l'a vu, privilégie l'écriture de la vérité, en vient progressivement à accepter les pseudonymes pour les raisons évoquées :

Je veux désormais que soient publiés en grand nombre dans chaque numéro de la revue des extraits de journaux personnels de différentes contributrices, des œuvres courtes et des fragments de réflexions personnelles. (...) Et si ces textes décrivent les émotions telles qu'elles ont été ressenties, sans passer par la forme d'un roman ou autre chose, cela ne pose aucun problème. (...) Néanmoins, celles qui éprouveraient quelques difficultés peuvent très bien prendre un pseudonyme. (Note de la rédaction, juin 1915)

En lisant ces mots, on comprend aisément pourquoi certains récits de soi à la première personne en viennent à s'enrichir de variations. Les exemples abondent en effet où la partie centrale, écrite à la première personne, est précédée ou suivie d'une narration d'une tout autre tonalité qui vient en quelque sorte encadrer le récit. 
Par exemple, Fuyu no owari 冬の終り [Fin d'hiver] (roman, mars 1915), de Yamada Kuniko 山田邦子, dépeint, à la première personne, les différentes étapes du cheminement intérieur de l'héroïne qui, en quête d'un Dieu véritable, se tourne vers le christianisme et endure les tourments du doute et l'angoisse de la mort avant d'entrevoir enfin un rayon de lumière. Mais, à côté du titre de ce roman, Fin d'hiver, est ajoutée un sous-titre : "Histoire qui m’a été contée par une femme nommée N. C. ». Dans Junjitsu no tomo 旬日の友 [Amies de dix jours] (roman, mars 1915) de Sugawara Hatsu 菅 原初, la narratrice se présente tout d'abord comme une amie de « $\mathrm{P}$. » dans le préambule, puis la forme du récit change radicalement après qu'elle a précisé que " pour une plus grande efficacité dans la représentation, P. s'exprimera désormais à la première personne ". Commence ensuite le récit des amours lesbiens de deux femmes P. (la narratrice) et K. Si l'on pense au caractère scandaleux pour l'époque d'un récit portant sur des relations homosexuelles, on peut supposer que l'ajout dans le préambule d'une autre narratrice visait, en confiant la narration à un autre personnage que P., partie prenante dans cette affaire, à bien souligner qu'il ne s'agissait pas là de l'expérience de l'auteure en personne. De même, Okada Yachiyo, qui raconte dans Hatsukoi no nariyuki 初恋のなりゆき [Destin d'un premier amour] (roman, août 1915-fév. 1916) la naissance et la fin de son premier amour, fait précéder le corps du récit par un très long préambule : "Le premier amour (...) ah, écoutez moi." Après cette longue entrée en matière, la femme commença doucement à raconter ce qui suit... ». Hasegawa Shigure fait également précéder le récit Usuzumi iro 薄ずみいろ [Gris pâle] (roman, sept. 1915-janv. 1916), d'une préface de quatre pages en petits caractères. Il s'agit chaque fois soit de prendre la précaution de voiler le récit, en raison même de sa vérité, ou bien d'un procédé visant à feindre le vraisemblable, ce qui dans les deux cas ne fait que souligner encore l'importance de la norme prescrivant la vérité.

\section{Retour au « roman » et mise en doute de la « femme nouvelle»}

Si j'ai pu formuler ces deux hypothèses sur les raisons pour lesquelles les auteurs éprouvaient alors le besoin d'encadrer leur récit, c'est parce qu’à cette étape le seul contenu des textes ne permet plus de déterminer si les 
expériences relatées sont celles des auteures elles-mêmes. Un autre changement vient corroborer cette nouvelle orientation puisque la plus grande partie des textes publiés est dès lors répertoriée dans la table des matières comme "romans". La classification générique de Seitō respectait-elle le désir des collaboratrices, ou bien était-elle le fait de la rédactrice en chef ? Nous en ignorons les circonstances exactes, mais en tout cas, cette nouvelle tendance confirme indéniablement la transformation des normes d'écriture depuis les déclarations véhémentes de Raichō et de Noe refusant catégoriquement que leurs textes soient lus comme des romans.

On voit alors réapparaître des textes écrits à la troisième personne. Le texte de Yamanoi Mineko, Sabishiki kokoro 淋しき心 [Un Cœur solitaire] (sans précision de genre, avril 1915) en est un exemple. On y retrouve les mêmes personnages que dans Onna tomodachi 女友達 [Les Amies] (réflexions personnelles, mars 1915) de Kawada Yoshi 川田よし, lequel texte ayant été classé comme kansō, " réflexions personnelles ", devait relater des faits véridiques. Yamanoi et Kawada se connaissaient donc personnellement, et Yamanoi se serait inspirée de sa propre expérience, transposée sous la forme d'une narration à la troisième personne. Il convient de souligner ici les traits caractéristiques d'Un Cour solitaire, car bien que conçu dans l'idée de contredire Les Amies par une critique des personnages qui apparaissent dans cet essai, ce roman ne mène pas la contradiction à son terme, puisqu'au final la lettre exprimant les objections à l'encontre de l'essai de Kawada Yoshi ne sera pas envoyée ${ }^{13}$. De même, Hasegawa Shigure peint dans Ishi no onna 石のをんな [Une Femme stérile] (roman, mai 1915), récit sous la forme d'une narration à la troisième personne, une femme nommée Nanako qui, songeant à écrire une lettre à une autre femme, sans enfant comme elle, réfléchit aux divergences de leurs points de vue concernant les enfants. Mais l'écriture de cette lettre n'était pas le

13. Voir l'analyse critique de Tokunaga Natsuko 徳永夏子 sur Un Coeur solitaire dans «Seitō ni okeru shutai no kakō : shōsetsu o meguru keishiki to ōtōsei »『青鞜』における 主体の仮構——小説をめぐる形式と応答性 (Construction fictionnelle du sujet dans Seitō : forme et dialogisme dans le roman), communication faite lors de la deuxième journée d'étude de la Société d'étude sur la littérature moderne japonaise, printemps 2011, 29 mai 2011, Nihon daigaku. 
but ultime de ses réflexions puisque le roman s'achève sur cette remise en question :

Nanako se disait au fond d'elle-même qu'il n'était pas besoin de montrer à son amie cette lettre qu'elle voulait lui écrire, et d'ailleurs plutôt que de s'abêtir à la rédiger, mieux valait finalement y renoncer. (...) Dans le fond, même si cela pouvait être de quelque utilité pour la société, cela n'était pas d'un grand intérêt pour les questions féminines, etc.

Le retour au récit à la troisième personne rend possible l'expression de la réticence des héroönes à passer à l'acte et à exprimer leurs opinions. Comme pour Un cour solitaire, l'auteure d'Une femme stérile a recours à la forme épistolaire pour exprimer ses opinions à la première personne. Mais s'il s'agissait seulement de transmettre les pensées de la narratrice aux lectrices, la partie écrite à la première personne eut suff. La narration à la troisième personne greffée à ce récit à la première personne est donc un dispositif narratif permettant à l'auteure d'expliciter l'impossibilité même dans laquelle se trouve la narratrice d'exprimer entièrement son point de vue. Si Nanako est en proie à l'incertitude, comme le montre le passage cité ci-dessus, c'est précisément parce qu'elle doute que ses difficultés personnelles soient vraiment liées aux "questions féminines ".

Nous avons pu observer depuis Fin de l'hiver plusieurs exemples dans lesquels la partie principale, constituée d'un récit à la première personne, était encadrée par la narration d'un tiers. Si bien que ces œuvres, même si elles prennent la forme d'un récit à la première personne, sont de fait, grâce à l'effet de distance ménagé par l'ajout d'une narration à la troisième personne, très proches des récits à la troisième personne. Ainsi la norme prescrivant d'écrire de manière à ce que les lectrices comprennent que les faits vécus par la narratrice correspondent à l'expérience vécue de l'auteure se défait-elle peu à peu. Cette tendance se manifeste dans les œuvres dans lesquelles la partie encadrant le récit s'hypertrophie et où la distance prise avec les sentiments du personnage principal montre que l'auteure, au lieu de se concentrer sur l'affirmation de soi et de ses opinions, préfere exprimer les doutes de son personnage sur l'utilité même d'affirmer ses opinions. La question pour nous n'est pas ici de savoir si l'auteure écrit la vérité, mais d'observer comment des textes présentant des particularités narratives telles que la superposition de récits à la première personne et à la troisième personne en sont venus à être répertoriés comme des romans. Il me semble que 
si cette manière de répertorier les textes s'est imposée naturellement au sein de la revue, c'est parce qu'elle représentait désormais une piste de recherche importante pour le groupe Seito.

Nous avons vu, dans Une Femme stérile, que certaines auteures étaient conscientes du fait que leurs textes, en tant que réflexions, n'abondaient pas dans le sens des "questions féminines ». De la même manière, la narratrice de Gris pâle se qualifie de femme "à l'ancienne ", explicitant ainsi la distance prise avec l'image de la «femme nouvelle».

Si j'écris cela, ce n'est pas pour le montrer à quiconque. (...) On pourrait penser de moi que, de nos jours, mes idées vieillottes et ma façon de vivre sont bien pitoyables. (...) Pourquoi en suis-je arrivée à penser ainsi ? Comment suis-je devenue aussi conventionnelle, dénuée de toute passion? Je veux essayer de retracer les sentiments et les émotions qui ont fait de moi ce que je suis aujourd'hui.

Satō Kinko 佐藤欽子 dans Shiraha no ato 白刃の跡 [Les Traces de la lame nue] (roman, oct. 1915) va jusqu'à dire que personne ne peut la comprendre et rejette toute expérience partagée :

Parmi elles, aucune ne pouvait partager mes douleurs, mes angoisses et mes déchirements, aucune n'éprouvait exactement les mêmes émotions et sentiments que moi. (...) En fin de compte, y avait-il parmi ces femmes une seule qui ait une exacte conscience de soi et qui soit assez forte pour décider de vivre vraiment seule ? Elle en doutait fortement (...) Mais depuis quand ?

Cette " exacte conscience de soi » était précisément la condition proclamée par les fondatrices pour devenir une "femme nouvelle ». À partir de la cinquième année, l'attitude commune, même si elle n'est pas clairement explicitée, est une relative prise de distance avec Raichō et les fondatrices, ainsi qu'avec toutes celles qui voulaient représenter les femmes ${ }^{14}$. Ces propos ne traduisent bien sûr que des doutes à l'égard de l'affirmation de soi et ne permettent pas de tirer de conclusions sur la formation d'une orientation

14. À propos de la « difficulté d'écrire » venant de l'impossibilité de devenir une «femme nouvelle ", voir Iida Yūko (2002). Iida distingue formellement deux types d'écriture et considère que la narration à la première personne est une "confession ", tandis que la narration à la troisième personne n'en est pas une. Mon propos n'est pas ici d'examiner les effets formels du roman, mais d'analyser comment la représentation de soi constitue le roman comme genre. 
dissidente au sein de la revue. La conscience de l'absence de toute interlocutrice capable de comprendre ce que l'une ou l'autre ressent n'était d'ailleurs pas un moteur suffisant pour constituer un groupe d'opposition. De fait, il s'agissait moins d'une levée de boucliers que de la manifestation de l'idée qu'il était difficile d'apprécier toutes choses à l'aune des seuls critères du féminisme. C'est bien parce que ces textes exprimaient certaines tendances au sein même du groupe qu'ils méritent de figurer parmi les textes " dans le style de Seitō».

\section{Une attitude partagée : continuer à écrire}

Pourquoi finalement ces auteures ont-elles continué à écrire si elles ne prétendaient plus transmettre intégralement leurs opinions à travers leur narratrice ? Car de fait, loin de renoncer à écrire, elles étaient bien décidées à continuer de s'exprimer. Je suis tentée d'en déduire que ce qu'elle voulait donner à lire, c'est précisément leur volonté d'écrire et que c'est dans cette volonté même qu'elles plaçaient désormais la vérité de l'écriture.

Les normes d'écriture avaient donc progressivement évolué, depuis la norme prescrivant de retracer les faits réels tels qu'ils avaient été vraiment vécus, vers un désir d'authenticité et de fidélité à soi-même. Les auteures ont continué à utiliser le terme "vérité ", non parce qu'elles prétendaient épuiser la "vérité » objective de certains faits réels ou encore la "Vérité " universelle, mais pour désigner une attitude ou une modalité du rapport de soi à l'écriture.

En outre, on peut penser que la multiplication de textes nommé shūsaku 習作 (étude) n'était pas le signe d'un détachement envers les œuvres « achevées » et bien ficelées, mais plutôt la conséquence d'une préférence pour l'authenticité face à soi et une certaine réserve dans l'affirmation de ses idées. Makino Kimie 牧野君江, qui peint la vie intérieure d'un personnage misanthrope et maladif, intitule son roman "Étude», Shūsaku (roman, déc. 1914), pour indiquer qu'elle essaie de représenter la situation dans laquelle elle se trouve pendant qu'elle écrit. La Première Euvre de Chihara Yoshi, mentionnée précédemment, n'était pas véritablement une première œuvre puisqu'elle succédait de fait à Ai suru shi e 愛する師へ [À mon maitre vénéré] (roman, mars 1915). D’ailleurs, bien qu'À mon maître vénéré soit répertorié comme un roman, on peut supposer, vu sa forme épistolaire, que cette classification 
ne respectait pas les intentions de l'auteure qui va continuer dans Première CEuvre à exprimer le processus d'écriture en soi. Si l'attention se porte sur l'acte d'écrire en soi, il n'est pas besoin de conclure le texte.

Vers la cinquième année, la tendance est ainsi à l'allongement des textes, sans pour autant qu'ils conduisent à un dénouement qui viendrait clore le récit des événements. Gris pâle de Hasegawa Shigure publié en feuilleton en quatre fois, Premier Amour d'Okada Yachiyo publié en six fois, Première Euvre de Chihara Yoshi publié en quatre fois, atteignent 120 pages chacun. Ces quelques cas mis à part, pour Seitō, cette longueur est exceptionnelle. Gris pâle, par exemple, s'achève brutalement sur ces mots : "J'ai toujours la rage d'écrire ce que je ressens, si une autre occasion se présente, je m'y consacrerai puisque ce que j'écris tant bien que mal a l'air de plaire " (janv. 1916). De même Première Euvre s'interrompt après le quatrième épisode paru dans le numéro de juillet 1915, alors que la mention "à suivre " ajoutée en fin du texte laissait attendre une suite. Dans les deux cas, un préambule annonce un retour sur soi avec des questions du genre "pourquoi suis-je devenue celle que je suis ? ", ou encore " pourquoi un tel événement s'est-il produit? ", mais les deux textes ne proposent ni dénouement ni quelque réponse que ce soit, et ne reviennent pas non plus au présent de la narration, à l'origine de l'évocation. La rétrospection n'a pas pour but l'explication des relations de causes à effets entre présent et passé, elle est une plongée dans le passé qui fait resurgir les émotions telles qu'elles ont été ressenties sans viser une reconstitution logique et un dénouement. La fin reste ouverte.

On devine dans Première Euvre que le pasteur a des sentiments trop romantiques, c'est pourquoi lorsqu'il envisage de s'enfuir avec elle, Y. se montre réticente. Elle observe avec une froide lucidité ce pasteur qui cherche à confier sa solitude à une personne de l'autre sexe à travers un long discours littéraire, et se dit :

"Lui aussi s'approche de moi avec une arrière-pensée. (...) Mais s'il a quelque arrière-pensée à l'égard de son interlocutrice, je dois avouer que moi aussi j'ai une petite idée derrière la tête; je ne cherche qu’à l'amadouer pour qu'il m'attire dans ses filets. " (mai 1915).

Telle est la dynamique interne qui pousse Y. à se transformer en femme d'action. En tant qu'observatrice, elle veut savoir jusqu'où la mènera cette relation. 
Pourtant, en poursuivant ses relations y compris épistolaires avec cet homme, Y. semble tout faire pour s'enfoncer dans une impasse. Bien qu'elle soit convaincue que les hommes sont plus sentimentaux que les femmes, elle écrit dans une lettre : "Je sens irrémédiablement la force irrésistible du destin qui va s'abattre sur moi d'une façon ou d'une autre. " Sans chercher à éviter l'approfondissement de cette liaison amoureuse, elle n'est pas sans comprendre que son attitude ambigüe est aussi un stratagème pour mieux séduire le pasteur. Un jour qu'elle était avec lui, le bruit assourdissant d'un train lui fait se souvenir du Triomphe de la mort de D'Annunzio, et l'air " distrait ", elle semble perdre la distance qui lui permettait de s'observer de l'extérieur. On ne saurait se contenter de constater un changement d'état mental de l'héroïne ou de penser que l'auteure choisit de se focaliser sur son personnage un temps follement amoureuse du pasteur. Tout se passe plutôt comme si Y., qui d'emblée se méfiait " des gens ignares à l'esprit obtus qui s'assemblent, s'inquiètent inutilement, fomentent de vains stratagèmes, font grand tapage... " et affichait tout au long du roman un détachement vis-à-vis des relations mondaines, nous était dépeinte comme un personnage vacillant.

On peut se demander si cela ne révèlerait pas un conflit interne de l'écrivain partagé entre un désir d'analyse et d'explication des événements vécus et un désir d'écriture sans qu'aucun dénouement ne soit prévu au préalable. Notons que la narratrice, pourtant neutre en tant que narratrice à la troisième personne, désigne la protagoniste "Y. san " avec respect et que cette initiale est aussi celle de l'auteure. De plus, le personnage de Première Euvre n'étant pas écrivain, le titre se réfère naturellement à la première œuvre de l'auteure elle-même. Autrement dit, on peut penser que l'auteure se peignait elle-même avec ironie, et que son refus de clore le roman sur l'heureux dénouement attendu par tous ne serait pas en soi un dispositif narratif lui permettant de préserver l'essence même de cette Première CEuvre en tant que processus d'écriture inachevé. L'interruption soudaine de cette œuvre nous conduit à penser que si ce récit ne débouche sur aucun raisonnement ou aucune interprétation, c'est parce que c'est l'exigence même d'ouverture au temps qui met brutalement fin au roman. Autrement dit, le récit est délibérément suspendu, laissé inachevé. Ceci n'est qu'un exemple parmi d'autres, mais force est de constater que, de même que la réalité est toujours un processus en cours, les romans sans dénouement se multiplient. 


\section{En guise de conclusion}

Comment au final évaluer cet ensemble de textes ? À partir de la cinquième année, la conscience du fait que chaque femme a une personnalité propre et ne peut s'identifier à un ensemble de femmes toutes identiques s'impose au sein de Seitō. Bien que ce phénomène ait pu être considéré comme un approfondissement du féminisme en soi, il ne s'agissait là encore que d'une prise de conscience individuelle. L'obtention de résultats politiques se fera attendre encore longtemps et la revue Seitō s'éteindra avant de voir ces résultats.

C'est pourquoi ces textes relèvent malgré tout davantage du champ littéraire que du champ politique. Depuis la polémique entre art et vie réelle ${ }^{15}$ des années 1907-1912, les écrivains masculins, avec des postures diverses, débattaient inlassablement de la représentation de soi et de la relation entre ce soi, conçu comme matériau d'observation, et les différentes formes d'exercices de l'objectivisation. L'attitude consistant à privilégier le processus d'écriture en tant que tel prit un nouvel essor durant l'ère Taishō qui vouait un culte particulier à l'instruction (kyōyō shugi 教養主義). Pendant les années 1910-1920, la pratique d'écriture consistant à se peindre soi-même à travers des personnages romanesques se répandit largement. Je pense que les femmes écrivains de Seitō, tout en cherchant à inventer d'autres chemins que ceux expérimentés par leurs homologues masculins, ont finalement accompli un parcours analogue. Elles ne lisaient bien évidemment pas que leur propre revue et étaient elles aussi sensibles à l'air du temps. On ne peut ignorer l'influence des problématiques de l'époque et des avancées littéraires sur leurs écrits, mais il est difficile de savoir quelles œuvres, quels auteurs critiques masculins elles lisaient plus particulièrement et d'en mesurer l'impact sur elles. J'ai voulu dans cet article prendre pour objet d'étude le territoire exclusivement formé par Seitō, afin d'examiner à l'intérieur de cet espace ainsi délimité la dynamique et les différents enjeux du moment et leur incidence sur l'évolution des dispositifs textuels et de l'écriture romanesque.

15. Il s'agit d'une des polémiques les plus importantes dans la définition du courant dit naturaliste, qui impliqua des auteurs connus, comme Shimamura Hōgetsu 島村抱月, Tayama Katai 田山花袋 et Iwano Hōmei 岩野泡鳴 [N.D.E.]. 
Au terme de cette analyse qui démontre combien il est difficile de préciser la nature exacte des relations entre littératures féminine et masculine produites à la même époque, on peut se demander s'il ne serait pas plus pertinent d'examiner dans quelle mesure ces différences ne proviendraient pas finalement aussi des lieux qui accueillent les œuvres des hommes et des femmes. Pour que des romans qui décrivent les états d'âmes d'un auteur en proie aux affres de l'écriture remportent du succès, il fallait que des informations sur l'écrivain en question circulent largement auprès du lectorat et attisent sa curiosité. Nul doute que les auteurs qui bénéficiaient d'un tel lieu de diffusion d'informations complétant les faits décrits à l'intérieur de leurs romans en tiraient un grand avantage. Sur ce point, on ne saurait douter de l'importance des cénacles littéraires masculins faisant office de caisses de résonance et propulsant les affaires personnelles sur les devants de la scène littéraire, alors que dans le même temps de telles affaires touchant les femmes écrivains n'étaient connues que par un cercle restreint de personnes. Cela expliquerait pourquoi bien que les femmes aient suivi des chemins d'expérimentation romanesque analogues à ceux des auteurs masculins, elles n'ont pas rencontré le même succès. Mais il faut signaler, au moins au niveau du contenu, l'émergence d'une situation nouvelle dans le monde littéraire : il est désormais difficile de mettre à part la création féminine en tant que genre littéraire spécifique. Mes travaux actuels tentent de développer ce point et d'examiner de plus près les occasions et les lieux dans lesquels les femmes écrivains jouèrent ou continuent de jouer un rôle de premier plan.

Traduit du japonais par Brigitte Lefevre (université Lille 3) 


\section{Bibliographie}

IIDA Yūko 飯田祐子 2002

«"Katarinikusa" to yomareru koto :

Sugimoto Masao no shōsetsu »

〈語りにくさ〉と読まれること——

杉本正生の小説 (De la difficulté d'écrire

et d'être lu : les romans de Sugimoto

Masao), in Seitō to iu ba. Bungaku, jendā,

《atarashii onna»『青靼』という場

一文学・ジェンダー・〈新しい女〉

(Seitō comme lieu. Littérature, genre, " femme nouvelle »), Tokyo,

Shinwasha 森話社, pp. 53-98.

KITADA Sachie 北田幸恵1998

«Gaitō ni deta onnatachi no koe :

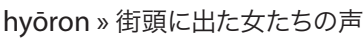

(Voix de femmes sorties dans la rue), in Shin feminizumu hihyō no kai 新・フェミニズム批評の会 (Association pour une nouvelle critique féministe) (éd.), Seitō o yomu『青鞜』を読む (Lire Seitō), Tokyo, Gakugei shorin 學藝書林, pp. 176-201.

MIZUTA Noriko 水田宗子1993

Monogatari to han-monogatari no fūkei 物語と反物語の風景 (Panorama des récits et des non récits), Tokyo, Tabata shoten 田畑書店.

ODAIRA Maiko 小平麻衣子 2008 Onna ga onna o enjiru. Bungaku, yokubō, shōhi 女が女を演じる—— 文学・欲望·消費 (La femme dans le rôle de la femme. Littérature, désir et consommation), Tokyo, Shin.yōsha 新曜社.
Raichō kenkyūkai らいてう研究会 (Société d'étude sur Raichō) 2001 Seitō jinbutsu jiten. Hyakujū nin no gunzō 『青鞜』人物事典—110人の群像 (Dictionnaire des femmes de Seitō. 110 portraits), Tokyo,Taishūkan shoten 大修館書店.

Shin feminizumu hihyō no kai 新・フェミニズム批評の会

(Association pour une nouvelle critique féministe) (éd.) 1998 Seitō o yomu『青鞜』を読む (Lire Seitō), Tokyo, Gakugei shorin 學藝書林.

TOKUNAGA Natsuko 徳永夏子 2010 "Seitō ni okeru jikogatari no henyō : tekusuto ni yoru genjitsu to no sesshoku »『青鞜』における自己語りの 変容—テクストによる現実との接触 (Métamorphoses du discours de soi dans Seitō : Le texte comme moyen d'accès au réel), Nihon bungaku 日本文学, 59 (9) : 31-41. 
